\title{
ESTIMACIÓN DE LA HUMEDAD SUPERFICIAL DEL SUELO MEDIANTE DATOS SAR EN BANDA $\mathrm{X}$
}

\author{
Héctor Salgado ${ }^{1,2}$, Stella Maris Zabala ${ }^{1}$, Catalina Romay ${ }^{1}$, Monique Bernier ${ }^{3}$, Andres Jacome ${ }^{3}$, Karem \\ Chokmani $^{3}$
}

\author{
${ }^{1}$ Riego y Drenaje, Facultad de Agronomía de Buenos Aires, Buenos Aires, Argentina \\ ${ }^{2}$ Meteorología, Servicio de Hidrografía Naval, Ministerio de Defensa, Argentina \\ ${ }^{3}$ Institut National de la Recherche Scientifique, Université du Québec, Québec, Canada
}

(Manuscrito recibido el 1 de octubre de 2019, en su versión final el 4 de febrero de 2020)

\section{RESUMEN}

El objetivo de este estudio es la estimación y el mapeo de la humedad del suelo a partir de imágenes Cosmo-Skymed (Banda X), adquiridas sobre una cuenca agrícola situada en el centro de la provincia de Buenos Aires, Argentina (Latitud: $37^{\circ} \mathrm{S}$; Longitud: $60^{\circ} \mathrm{W}$ aproximadamente). Se emplea una aproximación empírica, mediante correlación entre el coeficiente de retrodispersión $\left(\sigma^{0}\right)$ y las mediciones de humedad del suelo (HS), obtenidas simultáneamente con el pasaje satelital. La humedad volumétrica superficial $(0$ a $10 \mathrm{~cm}$ ) del suelo fue medida mediante el método gravimétrico en 15 parcelas agrícolas (sitios). La relación lineal entre la retrodispersión de la señal radar $\left(\sigma_{0}\right)$ y las mediciones de $H S\left[H S(\%)=b+a \cdot \sigma^{0}(d B)\right]$ presenta diversos coeficientes y ajustes $\left(r^{2}\right)$, dependiendo de la dirección orbital y la polarización (desde $r^{2}=0,28$ para datos VV Ascendente hasta $r^{2}=0,69$ para HH Descendente). Aplicando las ecuaciones correspondientes, las imágenes se transformaron de $\sigma^{0}$ a $H S(\%)$, obteniendo los mapas de HS (para cada polarización y tipo de órbita). Se realizó una validación cruzada entre datos de HS medida vs. estimada, calculando el Error Cuadrático Medio (RMSE) y el Error Medio Cuadrático Normalizado (NMSE). Los mejores resultados se obtuvieron con las imágenes Descendentes, con 6,3\% RMSE $<6,9 \%$ y $1,6<$ NMSE $<2,0 \%$. Se comparan los resultados con otras experiencias, y se plantean expectativas futuras. Palabras clave: radar, Cosmo-Skymed, humedad del suelo, abordaje empírico, cuenca agrícola.

\section{SURFACE SOIL MOISTURE ESTIMATION WITH X BAND SAR DATA}

\begin{abstract}
The aim of this study is to estimate and map soil moisture (SM) from Cosmo-Skymed (X band) images acquired over an agricultural basin located in the center of the province of Buenos Aires, Argentina (Latitude: $37^{\circ} \mathrm{S}$; Longitude: $60^{\circ} \mathrm{W}$ approximately). The adopted approach is empirical, with a correlation between backscattering coefficient $\left(\sigma^{0}\right)$ and soil moisture (SM) measures, obtained simultaneously with a satellite pass. Surface $(0$ to $10 \mathrm{~cm})$ soil moisture was measured by means of the gravimetric method in 15 agricultural parcels (sites).
\end{abstract}


The linear relation between radar signal backscattering $\left(\sigma^{0}\right)$ and field measured SM $\left[S M(\%)=b+a \cdot \sigma^{0}(d B)\right]$ shows diverse coefficients and adjustments (r2), depending on orbit direction and polarization (from $r^{2}=0,28$ for $\mathrm{VV}$ Ascending to $r^{2}=0,69$ for HH Descending). Using corresponding relation equations, images were translated from $\sigma_{0}$ into SM (\%), obtaining the SM maps (for each polarization and orbit pass). Cross-validation between measured and calculated SM was performed, computing the Root Mean Square Error (RMSE) and Normalized Mean Square Error (NMSE). Better results were obtained from Descending images, with $6,3 \%<$ RMSE $<6,9 \%$ and $1,6<\mathrm{NMSE}<2,0 \%)$. Results are compared with other experiences, and future expectations are outlined.

Keywords: radar, Cosmo-Skymed, soil moisture, empirical approach, agricultural basin.

\section{INTRODUCCIÓN}

La humedad superficial del suelo (HS), indicadora principalmente del agua en estado líquido en la zona no saturada, juega un rol decisivo en el ciclo hidrológico, participando activamente en los flujos del $\mathrm{C}$ y de energía en la interfase suelo-vegetación-atmósfera.

$\mathrm{Su}$ estimación precisa, considerando su amplia variabilidad espacial y temporal, resulta fundamental para estudios y tareas ambientales y agrícolas, contribuyendo, entre otras aplicaciones, al monitoreo del estado hídrico de suelos en producción agrícola (Moran et al., 2004).

Aun cuando, desde los años 70, se ha estimado indirectamente la HS mediante sensores satelitales ópticos y térmicos, recién a partir de los 90 la teledetección satelital de microondas ha demostrado que puede medirse directamente, de manera rutinaria, con diferente resolución espacial y frecuencia temporal (Wang y $\mathrm{Qu}$, 2009).

Los sistemas activos, como el Radar de Apertura Sintética (Synthetic Aperture Radar SAR), tienen una amplia cobertura espacial (franjas de 30 a $100 \mathrm{~km}$ de ancho), con una frecuencia temporal mensual, alcanzan una resolución espacial entre 1 y 30 m, y pueden captar imágenes independientemente de las condiciones lumínicas (tanto de día como de noche) y de nubosidad. Los radares satelitarios emiten, de manera ortogonal a su trayectoria y oblicua respecto a la superficie terrestre, una onda electromagnética, que alcanza el suelo y sufre dispersión de diverso tipo. Parte de la señal retorna al sensor, en forma de energía retrodispersada, y su magnitud se expresa mediante el coeficiente de retrodispersión " $\sigma^{0}$ ", siendo su unidad de medida el decibel (dB).

La capacidad de los SAR para detectar la humedad en el suelo radica en que la retrodispersión captada depende de las características geométricas y físico-químicas de la escena. En particular, $\sigma^{0}$ es función de la constante dieléctrica " $\varepsilon$ " del suelo, la cual se incrementa fuertemente al aumentar su contenido de agua (Ulaby, 1974; Schmugge y Wang, 1980).

La intensidad de la respuesta a la señal radar depende de factores, tanto de la escena o blanco (geometría y rugosidad, HS), como del sistema sensor (polarización, longitud de onda " $\lambda$ ", ángulo de incidencia " $\phi$ "), y de la relación entre el terreno y la dirección de la trayectoria satelital (órbita ascendente o descendente).

En general, el $\sigma^{0}$ es función de la HS de un estrato, cuyo espesor depende de las propiedades de penetración en el suelo para esas longitudes de onda $(\lambda)$. También depende de la rugosidad del suelo y de la vegetación. Las variaciones de $\sigma^{0}$ con la HS, rugosidad de superficie, ángulo de incidencia, longitud de onda y textura del suelo 
vienen siendo estudiados desde hace más de tres décadas (Ulaby y Dobson, 1988).

En lo referente a la polarización de un radar, se caracteriza por la de su onda emitida y por la componente medida de retorno. Por ejemplo, VV (Vertical-Vertical) significa que emite una onda polarizada verticalmente (en realidad su campo eléctrico) y mide la componente vertical de la onda recibida. Los antecedentes bibliográficos (Ulaby y Batlivala, 1976; Brisco et al., 2008) señalan que, para suelos desnudos o con baja cobertura (menos de $15 \mathrm{~cm}$ de altura), la polarización $\mathrm{HH}$ es mejor que la $\mathrm{VV}$ para estimar la HS. En efecto, la polarización VV presenta una retrodispersión general más baja, produciendo una menor relación señal/ruido, lo cual reduce la sensibilidad a la humedad del suelo. La consideración de mejor polarización HH para estimación de HS fue comprobada mediante análisis de sensibilidad utilizando modelos teóricos de dispersión de superficie por Touré et al. (1994).

En cuanto a la $\lambda$, la penetración en el suelo es mayor, cuanto mayor sea $\lambda$. En general, a constancia de los demás factores del radar (ángulo de incidencia, polarización, banda), la penetración es una fracción de la longitud de onda (de $1 / 2$ a $1 / 3$ ). O sea, un fino estrato superficial de 1 a $1,5 \mathrm{~cm}$ para la Banda X (Ulaby et al., 1996).

Por otra parte, el comportamiento (reflexión y penetración) de una onda radar sobre una superficie depende del ángulo entre la dirección del haz y la normal a la superficie (ángulo de incidencia " $\phi ")$. Como regla general, la penetración de las ondas es tanto más profunda cuanto más pequeño es $\phi$.

Las imágenes SAR COSMO-Skymed, utilizadas en este trabajo, corresponden a la Banda $\mathrm{X}(\lambda=3,1 \mathrm{~cm} ; f=9,6 \mathrm{GHz})$, modo Ping-Pong, aportando datos en 2 polarizaciones: HH (Horizontal-Horizontal) y VV.

La interpretación de la señal SAR se basa en la comprensión de la relación entre $\sigma^{0} \mathrm{y}$ las propiedades dieléctricas y geométricas del blanco. Para definir esta relación se desarrollaron distintas aproximaciones. Abordajes estadísticos, con diversos algoritmos (Bayesiano, Redes Neuronales, Minimización Nelder-Mead, regresiones, etc.), fueron tratados por Santi et al., 2005. En el presente trabajo se utilizó la regresión entre $\sigma^{0}$ y parámetros geofísicos (humedad, rugosidad, etc.), la cual se manifiesta de manera aproximadamente lineal, para el rango de valores frecuentes de HS, en diversos estudios (Brisco et al., 1997; Santi et al., 2005; Aubert et al., 2011). En el caso de suelos desnudos, la relación se expresa por:

$$
\sigma^{0}(d B)=a \cdot H S(\%)+b
$$

donde " $b$ " (ordenada al origen) representa la contribución de la rugosidad de la superficie (por cobertura vegetal) y " $a$ " (pendiente) es la contribución de la HS y de la configuración radar.

La inversión de (1) permite la estimación de HS directamente a partir de la retrodispersión registrada en la imagen (Chanzy et al., 1994):

$$
H S(\%)=m+n \cdot \sigma^{0}(d B)
$$

Numerosos estudios sobre la estimación de la HS en diversos ambientes han sido recopilados por Engman (2000), Moran et al. (2004), Álvarez-Mozos y Crespo (2005), Dabrowska-Zielinska et al.(2005) y Wang y Qu (2009). Los mismos se detallan y comparan en la Sección "3) Resultados y Discusión" del presente trabajo.

En una zona productiva agrícola de la Pampa Argentina se obtuvieron buenos ajustes (coeficiente de determinación $r^{2}=0,68$; límites de confidencia $95 \%$ ) en la relación $\sigma^{0}$ vs. HS con sistemas en Banda C y $\phi=24 \check{z}$ (VV ERS y HH Radarsat), para condiciones en las cuales la cobertura vegetal no es densa y en función de la polarización de la señal (Salgado et al., 2001; Salgado, 2012).

El objetivo del presente trabajo es medir el grado de relación entre HS, captada simultáneamente 
con el pasaje satelital en una red de medición implementada "ad hoc", y $\sigma^{0}$, registrado con imágenes SAR en banda X, y la confección de mapas resultantes de dicha relación.

\section{MATERIALES Y MÉTODOS}

\subsection{Zona piloto y sitios de medición de HS}

Dada la naturaleza hidrológica del estudio, se definió una cuenca hídrica como marco de referencia física del mismo. Se seleccionó la Cuenca del Arroyo Pablo Acosta (Fig.1), afluente del Arroyo de Los Huesos, ubicada en el centro de la provincia de Buenos Aires, Argentina (Latitud $37^{\circ} \mathrm{S}$, Longitud $\left.60^{\circ} \mathrm{W}\right)$. Se encuentra en la región serrana de la denominada Pampa Húmeda, cuya principal actividad económica es la agropecuaria.

Los suelos predominantes corresponden a la siguiente taxonomía (US Soil Survey Staff, 1975): Orden: Molisoles; Suborden: Udoles; Grandes Grupos: Argiudoles y Hapludoles. Los Argiudoles son el gran Grupo más representativo de los Udoles. Su perfil típico muestra la sucesión de horizontes "A1 - B1 - B2t - B3 - C", con alto contenido de materia orgánica en el $\mathrm{A}$, y arcilla en el B2t. Las clases texturales predominantes son: franca y franca arcillo-limoso.

Se realizaron campañas de medición de la HS en 15 sitios (Fig.1), de manera simultánea con el pasaje del satélite. Se determinó la densidad aparente y la humedad volumétrica superficial (0 a $10 \mathrm{~cm})$ del suelo, mediante muestreo con barreno y pesada, siguiendo el método gravimétrico tradicional, el cual es considerado "patrón" para calibrar el resto de los métodos indirectos. A su vez, es el método estándar para comparar con mediciones en red a nivel global (Robock et al., 2000).

Los sitios de medición fueron seleccionados de acuerdo al siguiente criterio:

1. Que sean representativos de reales condiciones de cultivos típicos en la zona.
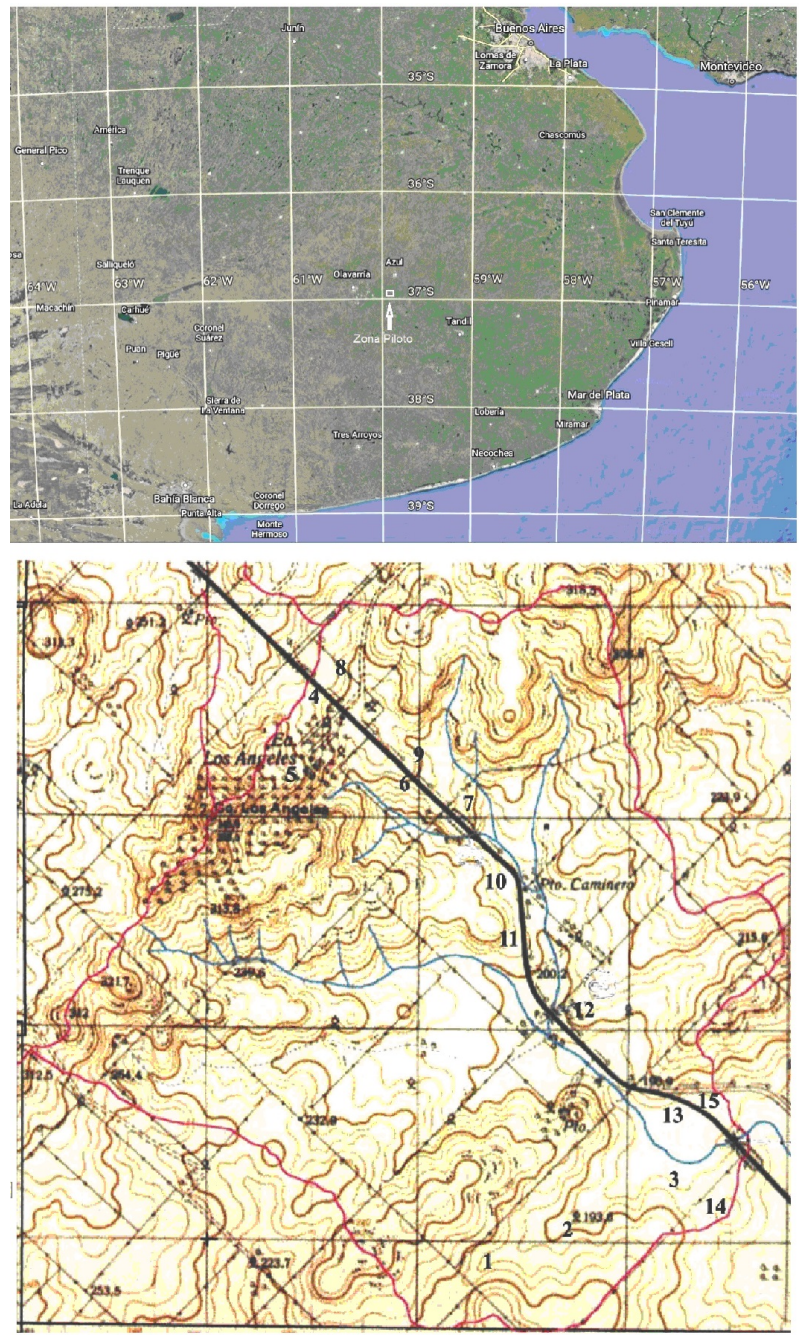

Figure 1: Ubicación geográfica de la Zona Piloto, y detalle de la Cuenca del A. Los Ángeles (marcada con línea roja) y sitios de medición de HS (números arábigos).

En el interior de la parcela, alejados de los alambrados, ya que ahí los suelos no son laboreados, y por consiguiente su balance hídrico es diferente.

2. Que las coberturas vegetales correspondan a la más amplia variedad de las frecuentes (especies, sistemas de cultivo, etc.), teniendo en cuenta las rotaciones propias de la zona.

3. Que sean fácilmente accesibles, en las diversas condiciones ambientales, que se presentan a lo largo del año. 
Los resultados de las medias de las 3 repeticiones en cada sitio, para las fechas de pasajes satelitales, se muestran en Tabla I.

Como puede observarse en la última columna de Tabla I, al 4 enero 2012 le correspondió la situación de suelo más seco (15,6\% en promedio), mientras que al 26 octubre 2012, la mayor HS (36,50\% en promedio).

También se han registrado, para cada sitio y fecha, datos sobre la cobertura vegetal, que influye en la retrodispersión registrada. En promedio, la cobertura se muestra en Tabla II.

Dadas las características actuales de la agricultura, con predominio de sistemas de "siembra directa", prácticamente no hay situaciones de suelos "desnudos" en ningún momento del ciclo. Las condiciones más cercanas son las de rastrojos pastoreados, que presentan la menor rugosidad, mientras que coberturas vegetales más densas están dadas por cultivos (maíz, girasol, soja, etc.) en pleno desarrollo vegetativo.

\subsection{Imágenes COSMO y procesamiento digital}

Se emplearon imágenes SAR de la Misión italiana COSMO-Skymed (COnstellation of small Satellites for the Mediterranean basin Observation), en banda $\mathrm{X}(9,6 \mathrm{GHz})$, cuyo horario de paso es a las 09.50 PM en el modo Descendente (Desc), y a las 10.40 AM en el Ascendente (Asc) (Tabla III).

Mediante el software libre NEST 4C (European Space Agency), se realizaron las calibraciones radiométricas, correcciones geométricas, y transformación de potencia a amplitud en decibeles (dB). Dado que se emplearon imágenes COSMO con un Nivel de Proceso Geocodificado 1B (Gec), se saltearon los pasos de vista múltiple (Multilook) y de corrección del terreno (Terrain Correction).

Se correlacionaron los valores de $\sigma^{0}(\mathrm{~dB})$ con los correspondientes de HS( \%) mediante regresión lineal simple, obteniendo las debidas funciones, las cuales fueron utilizadas para establecer la correspondencia y la tabla de colores de los mapas de HS. Para disminuir la influencia del ruido "speckle", propio de las imágenes SAR, el valor de $\sigma^{0}$ se obtuvo del promedio (extraído de la imagen en potencia) de una ventana de $3 \times 3$ píxeles, centrada en las coordenadas del sitio de muestreo.

Los mapas se focalizaron en la zona piloto, por lo cual se generó una subimagen, limitada por un rango en Latitud ( $\left.37^{\circ} 06^{\prime} \mathrm{S}-37^{\circ} 10^{\prime} \mathrm{S}\right)$ y Longitud ( $\left.59^{\circ} 45^{\prime} \mathrm{W}-59^{\circ} 39^{\prime} \mathrm{W}\right)$ y se empleó la Proyección cartográfica Gauss-Krüger y Elipsoide de Referencia Internacional 1924.

Los valores de HS estimados se compararon con los medidos a campo mediante el Error Cuadrático Medio (RMSE, por su sigla en inglés) y el Error Cuadrático Medio Normalizado (NMSE, por su sigla en inglés), expresados mediante las fórmulas siguientes:

$$
\begin{aligned}
R M S E & =\sqrt{\frac{1}{n} \sum_{i=1}^{n}\left(x e_{i}-x o_{i}\right)^{2}} \\
N M S E & =\frac{\sum_{i=1}^{n}\left(x e_{i}-x o_{i}\right)^{2}}{\overline{x e_{i}}-\overline{x o_{i}}}
\end{aligned}
$$

donde $x e_{i} \quad$ y $x o_{i}$ son los valores estimados (calculados) y observados (medidos) respectivamente, $\overline{x e_{i}}$ es el promedio de la HS estimada, $\overline{x o_{i}}$ es el promedio de la HS observada, y $n$ es el número de pares de datos.

\section{RESULTADOS Y DISCUSION}

\subsection{Relación HS - retrodispersión}

Del análisis del conjunto de datos $(n=112)$, se observa en Fig. 2 una buena intensidad de relación entre las variables HS y $\sigma^{0}$, expresada a través del coeficiente de correlación de Pearson $(r=0,71)$ y un ajuste aceptable al modelo de regresión lineal simple $\left(r^{2}=0,51\right)$, dada la amplia diversidad de condiciones (coberturas, órbitas, polarizaciones) registradas. El coeficiente de determinación $\left(r^{2}\right)$ indica que el $51 \%$ de la 


\begin{tabular}{|c|c|c|c|c|c|c|c|c|c|c|c|c|c|c|c|}
\hline & $\mathbf{1}$ & $\mathbf{2}$ & $\mathbf{3}$ & $\mathbf{4}$ & $\mathbf{6}$ & $\mathbf{7}$ & $\mathbf{8}$ & $\mathbf{9}$ & $\mathbf{1 0}$ & $\mathbf{1 1}$ & $\mathbf{1 2}$ & $\mathbf{1 3}$ & $\mathbf{1 4}$ & $\mathbf{1 5}$ & Media \\
\hline 4-Ene-12 & 11.3 & 15.0 & 15.4 & 17.0 & 23.5 & 13.7 & 14.5 & 13.7 & 16.0 & 16.0 & 11.2 & 19.4 & 14.1 & 18.0 & $\mathbf{1 5 . 6}$ \\
\hline 2-May-12 & 22.4 & 31.8 & 22.1 & 38.0 & 32.8 & 31.9 & 33.9 & 35.4 & 33.8 & 34.2 & 30.7 & 36.5 & 28.7 & 35.7 & $\mathbf{3 2 . 0}$ \\
\hline 26-Oct-12 & 44.5 & 42.3 & 25.8 & 36.9 & 39.1 & 31.6 & 42.6 & 37.9 & 36.0 & 33.2 & 38.4 & 39.7 & 30.2 & 33.1 & $\mathbf{3 6 . 5}$ \\
\hline 19-Dic-12 & 30.1 & 33.7 & 29.3 & 30.5 & 31.9 & 31.8 & 32.8 & 41.1 & 31.1 & 26.7 & 26.8 & 33.5 & 30.3 & 34.3 & $\mathbf{3 1 . 7}$ \\
\hline
\end{tabular}

Table I: Mediciones de HS (\%) simultáneas con el pasaje satelital.

\begin{tabular}{|c|c|}
\hline Fecha & Cobertura \\
\hline 4-Ene-12 & Soja (60\%); Maíz (30\%); Rastrojo (10\%) \\
\hline 2-May-12 & Soja (35\%); Rastrojo (35\%); Maíz (20\%); Avena (10\%) \\
\hline 26-Oct-12 & Rastrojo (60\%); Avena (30\%); Colza (5\%); Trigo (5\%) \\
\hline 19-Dic-12 & Rastrojo (55\%); Soja (20\%); Maíz (20\%); Trigo (5\%) \\
\hline
\end{tabular}

Table II: Tipo y $\%$ de coberturas, en promedio, para cada fecha.

\begin{tabular}{|c|c|c|c|c|c|}
\hline Fecha & Orbita & $\begin{array}{c}\text { Ang. Inc. } \\
\boldsymbol{\phi}\left({ }^{\circ}\right)\end{array}$ & Modo & Polarización & $\begin{array}{c}\text { Pixel } \\
(\mathbf{m})\end{array}$ \\
\hline 04 Ene 12 & Desc. & 40 & Ping-Pong & HH - VV & 10 \\
\hline 02 May 12 & Asc. & 40 & Ping-Pong & HH - VV & 10 \\
\hline 26 Oct 12 & Desc & 40 & Ping-Pong & HH - VV & 10 \\
\hline 19 Dic 12 & Asc. & 40 & Ping-Pong & HH - VV & 10 \\
\hline
\end{tabular}

Table III: Imágenes COSMO-Skymed, con centro en: Latitud $37^{\circ} 08^{\prime} \mathrm{S}$; Longitud $59^{\circ} 42^{\prime} \mathrm{W}$.

variabilidad en HS es explicada por la variable independiente $\sigma^{0}$ en el modelo de regresión utilizado.

Siguiendo el criterio de los mínimos cuadrados se obtuvo la siguiente ecuación para la estimación de la recta del mejor ajuste a los datos:

$$
H S(\%)=58,076+1,7585 . \operatorname{sigma}^{0}(d B)
$$

donde la intersección $b_{o}=58,076$ representa el valor promedio de HS cuando $\sigma^{0}$ es igual a cero y la pendiente indica que un aumento unitario en $\sigma^{0}(\mathrm{~dB})$ representa un aumento del $1,7585 \%$ en HS.

La relación lineal significativa entre ambas variables se corrobora en el análisis de la varianza (ANOVA), a través del estadístico de prueba $\mathrm{t}$ con $n-2$ grados de libertad, tanto para el intercepto $\left(t_{\beta 0}=21,536\right)$ como para la pendiente $\left(t_{\beta 0}=10,662\right)$, con un nivel de significancia $\alpha \leq 0,05$ en ambos casos, y de la estimación del intervalo de confianza del $95 \%$ para la pendiente verdadera entre 1,365 y 1,988 .

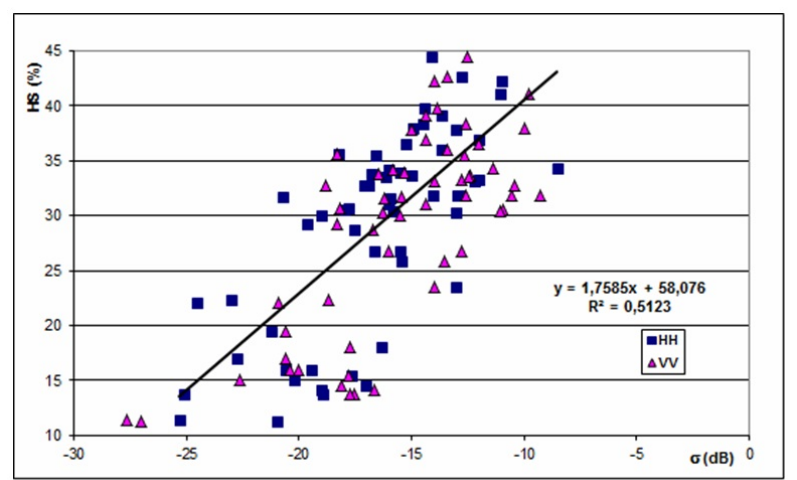

Figure 2: Relación general Humedad del Suelo "HS" vs. Retrodispersión de la Señal Radar " $\sigma$ ".

\begin{tabular}{|c|c|c|c|c|}
\hline Orbita y Polarización & Ord. al Origen & Pendiente & $\mathbf{r}^{\mathbf{2}}$ & $\mathbf{n}$ \\
\hline Asc. HH y VV & 42,0 & 0,66 & 0,31 & 56 \\
\hline Desc. HH y VV & 63,9 & 2,26 & 0,65 & 56 \\
\hline Desc. HH & 64,4 & 2,31 & 0,69 & 28 \\
\hline Desc. VV & 63,6 & 2,21 & 0,62 & 28 \\
\hline
\end{tabular}

Table IV: Parámetros de las rectas de ajuste.

De acuerdo a Brisco et al. (1997), la sensibilidad de la señal SAR a la HS es de $0,57 \mathrm{~dB} / \%$. Considerando que el ángulo de incidencia para todas las imágenes es de $40^{\circ}$, el resultado es comparable con los de Aubert et al.(2011), que obtuvieron una respuesta de $0,411 \mathrm{~dB} / \%$ con ángulos de incidencia bajos $\left(25^{\circ}\right)$ y de 0,323 $\mathrm{dB} / \%$ con mayores ángulos $\left(50^{\circ}\right)$, con imágenes TerraSAR-X (9,5 GHz; HH; pixel $1 \mathrm{~m})$ en la cuenca del Orgeval (Francia).

Desagregando el conjunto de datos según tipo de órbitas (Asc o Desc) y polarizaciones (HH o VV), las correlaciones se muestran en Tabla IV.

En síntesis, las funciones resultan totalmente distintas, según la dirección del pasaje orbital. El 
mejor ajuste $\left(r^{2}=0,65\right)$ se presenta con las Desc, lo cual se atribuye a que, en este caso, el ángulo entre el haz SAR y la dirección de los cultivos facilita la obtención de información del suelo, con menor influencia de la cobertura vegetal. A su vez, la polarización HH resulta levemente superior $\left(r^{2}=0,69\right)$ a la VV $\left(r^{2}=0,62\right)$. Esto era previsible, dado que la polarización $\mathrm{HH}$ es mejor que la VV, para estimar HS superficial, con escasa cobertura vegetal y ángulo de incidencia bajo (Ulaby y Batlivala, 1976). Con ángulos mayores (en este caso, $40^{\circ}$ ) aumenta la influencia del canopeo, como resumen Ulaby y Dobson (1988) y Brisco et al. (2008).

En general, la estimación de HS ha sido motivo de numerosos estudios con Banda C. En comparación con experimentos a escala de cuenca (Álvarez-Mozos y Crespo, 2005), el ajuste $\left(r^{2}=0,51\right)$ resulta aceptable, considerando los tipos de polarización y la diversidad de coberturas registradas en las fechas de las imágenes disponibles. Si se toma en cuenta sólo la polarización VV $\left(r^{2}=0,62\right)$, el ajuste no alcanza a otros trabajos, con coberturas agrícolas más homogéneas de cereales, como el Álvarez-Mozos y Crespo (2005), con valores de $r^{2}=0,87$ y pendiente $=4,7$. Cuanto más cultivos participen, mayor diversidad de canopeo y menor ajuste, como reportan Santi et al. (2005), que trabajaron con imágenes HH ASAR Envisat, con parcelas de trigo, cebada, alfalfa, pastura, maíz, poroto y remolacha azucarera, obteniendo un ajuste $r^{2}=0,4$ y pendiente $=2,5$ en promedio.

Dado que la relación HS vs. $\sigma^{0}$ está fuertemente influenciada por la rugosidad superficial de la vegetación, en Dabrowska-Zielinska et al. (2005) se propone asumir que la rugosidad se caracteriza por el Índice de Área Foliar "IAF". Así, en Dabrowska-Zielinska et al. (2005) se reporta un ajuste $r=0,86$ y pendiente $=3,2$ para cereales con $\mathrm{IAF}>3$, utilizando imágenes VV ERS. Con la misma polarización $\mathrm{VV}$, pero con imágenes ASAR Envisat en modo "Wide" (resolución-150m), Kong y Dorling (2005) obtuvieron un $r^{2}=0,32$ para campos de trigo. Por su parte, empleando imágenes HH Radarsat
S1 y S2, en Álvarez-Mozos y Crespo (2005) se obtuvo un ajuste discreto $\left(r^{2}=0,52\right.$; pendiente $=2,5$ ) para parcelas con cereales, lo cual mejora significativamente cuando se lo compacta $\left(r^{2}=\right.$ 0,75 ; pendiente $=3$ ).

Finalmente, en la misma zona piloto de la provincia de Buenos Aires, Argentina, Salgado (2012) reportó buenos resultados con Banda C y $\phi=24 \check{z}$ para lotes con trigo en emergencia, con mínima cobertura del suelo $\left(r^{2}=0,86\right.$; pendiente $=2$; polarización $\mathrm{HH}$ y $\mathrm{VV})$, y en estado vegetativo $\left(r^{2}=0,77\right.$; pendiente $=1$; $\mathrm{HH})$.

\subsection{Mapas de HS}

Aplicando las ecuaciones de las rectas de ajuste (presentes en la Tabla IV), se realizó el mapeo de HS para cada polarización y tipo de órbita. Se asignó una escala de colores con rangos de $5 \%$ de HS, desde $10 \%$ (naranja) hasta $50 \%$ (verde oscuro), pasando por tonalidades amarillas y verdosas. En Fig. 3 se presentan los mapas para imágenes Desc VV, cuya función es: $H S=$ $63,6+2,21 . \sigma^{0}$ (Tabla IV).

En los mapas se observa un predominio de colores amarillos para el 4ene12 (HS media $=15,6 \%$ ) y tonos verdosos para el 26 oct 12 (HS media = $36,5 \%$ ), como consta en Tabla I. Los cuerpos de agua superficiales (embalses, lagos, etc.) aparecen en negro (baja retrodispersión, por reflexión especular), y en rojo figuran elementos con alta retrodispersión (masa forestal, afloramientos rocosos, planta de silos, etc.).

\subsection{Validación cruzada del abordaje empírico}

El ajuste entre los valores de HS en imágenes Desc, calculados con la expresión $H S=63,9+$ $2,26 . \sigma^{0}$ (Tabla IV), y los medidos a campo se evaluó mediante las fórmulas RMSE (3) y NMSE (4), y se presenta en la Tabla V.

Los resultados son comparables con los obtenidos por Lievens y Verhoest (2012) (4,5\% < 

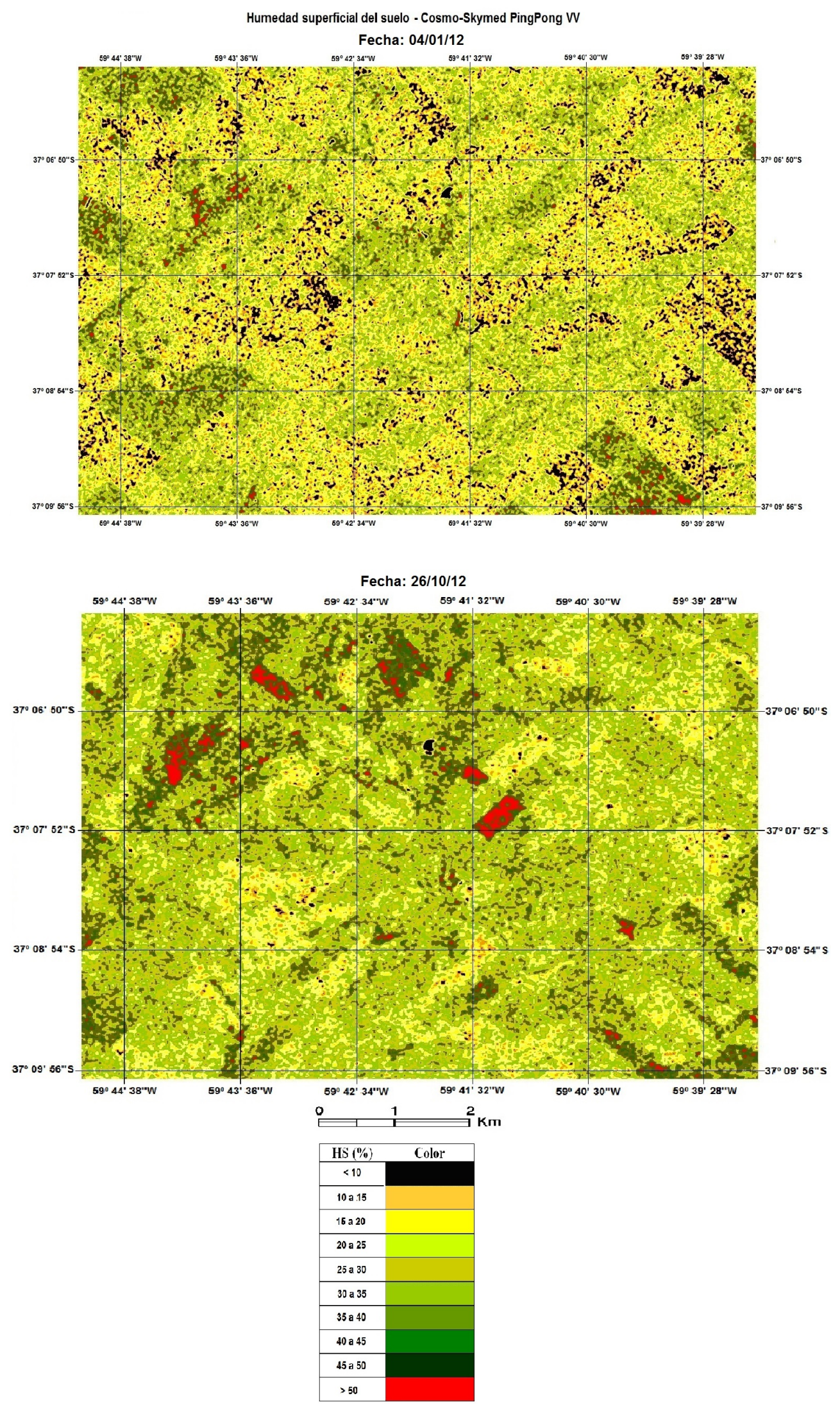

Figure 3: Mapas de HS para órbitas Desc, en fechas 4ene12 (seco) y $260 c t 12$ (húmedo). Los colores indican diferentes contenidos de humedad volumétrica, desde $<10$ a $>50 \%$. 


\begin{tabular}{|l|c|c|}
\hline & Pol. HH & Pol. VV \\
\hline RMSE & 6,31 & 6,97 \\
\hline NMSE & 1,62 & 2,03 \\
\hline
\end{tabular}

Table V: Errores: Cuadrático Medio (RMSE) y Medio Cuadrático Normalizado (NMSE).

$R M S E<9,8 \%$, según estado y cobertura de las parcelas), con imágenes Radarsat-2 en Banda C (5,3 GHz) en modo "Single Look Complex" (SLC), con cuadripolarización (HH-HV-VH-VV), haz "Fino" (FQ) y ángulos de incidencia del orden de $25^{\circ}$ en Flevoland, Holanda, trabajando con un modelo físico sobre distintas superficies agrícolas (con distintas rugosidades). La diferencia puede atribuirse, entre otros factores, a la mayor penetración en el suelo de la Banda C y al menor ángulo de incidencia.

\section{CONCLUSIONES}

Aunque es ampliamente conocido el empleo de imágenes SAR para la estimación de la HS, no hay muchos trabajos realizados con Banda X. Sin embargo, resulta muy útil para detección de elementos superficiales (rugosidad, follaje, etc.). Los cuerpos de agua han sido bien identificados con el ángulo de incidencia $\left(40^{\circ}\right)$ empleado.

La función general de regresión lineal obtenida $\left[H S(\%)=58,08+1,758 \cdot \sigma^{0}(d B)\right]$ muestra un ajuste discreto $\left(r^{2}=0,51\right)$, y deberá ser validada con mayor experimentación. La sensibilidad de la señal SAR respecto a la HS fue de $0,57 \mathrm{~dB} / \%$, y resulta comparable con las obtenidas en otros trabajos (entre 0,32 y 0,57 dB/\%), con bandas C y X, y distintos $\phi$.

El empleo de las relaciones HS vs. $\sigma^{0}$ permitió la generación de mapas de HS, que brindan su distribución espacial y pueden actualizarse con cada pasaje satelital.

Debido al abordaje empírico, tanto las funciones lineales como los mapas de HS resultan dependientes de:
- órbita (Asc. o Desc.) y su relación con direcciones predominantes de laboreo de las parcelas agrícolas

- ángulo de incidencia $\phi$

- cobertura vegetal (época del año)

La Red de Medición ha demostrado ser útil para provisión de datos simultáneos de HS en zonas bajo producción agrícola, para calibración y ajuste de actuales y futuras misiones satelitales.

Los resultados obtenidos alientan a continuar con estudios, usando mayor cantidad de imágenes (con ángulos de incidencia más bajos, polarizaciones directas y cruzadas, etc.), con vistas a su potencial uso complementario con imágenes SAR polarimétricas en banda L (de los satélites SAOCOM), en la Constelación del Sistema Ítalo Argentino de Satélites para la Gestión de Emergencias SIASGE.

Agradecimientos: Deseamos expresar nuestro agradecimiento al Téc. Marcelo Schwab, de la Facultad de Agronomía de la Universidad Nacional del Centro de la Pcia. de Bs.As., por su participación en el trabajo de campaña, y a la Ing. Claudia Carrascal, por su participación en el proceso inicial de las imágenes. También a la Comisión Nacional de Actividades Espaciales CONAE, institución que, a través de su Anuncio de Oportunidades, apoyó el Proyecto $30 / 11$, por el cual se obtuvieron las imágenes COSMO-Skymed y la capacitación para su uso.

\section{REFERENCIAS}

Álvarez-Mozos J. y Crespo J. J., 2005: Estimación de la humedad superficial del suelo en cuencas agrícolas por medio de imágenes de teledetección radar. Congreso Internacional Conjunto XVII Ingegraf - XV ADM Ingeniería Gráfica 2005, Sevilla, España, 1-3 junio 2005.

Aubert M., Baghdadi N., Zribi M., Douaoni A., Loumagne C., Baup F., El Hajj M. 
y Garrigues S., 2011: Characterization of the soil surface by TerraSAR X imagery. Remote Sensing of Environment, 115 (8), 2011, pp.1801-1810.

Brisco B., Pultz T., Brown R. y Gwyn H., 1997: Soil moisture estimation with RADARSAT. IGARSS'97, Ottawa, Canadá.

Brisco B., Touzi R., van der Sanden J.J., Charbonneau F., Pultz T.J. y D'Iorio M., 2008: Water resource applications with Radarsat-2 - a preview. International Journal of Digital Earth, 1:1, 2008, pp.130-147.

Chanzy, A., P. Bertuzzi y L. Bruckler, 1994: Estimation de la teneur en eau de la surface des sols nus a l'aide des mesures d'hyperfrequences actives. Dans S. Bonn (dir.), Télédétection de l'environnement dans l'espace francophone, Sainte-Foy, Canada, PUO/ACCT, pp.55-74.

Dabrowska-Zielinska K., Inoue Y., Kowalik W. y Gruszczynska M., 2005: Modelling of crop and soil parameters using $\mathrm{C}$ and $\mathrm{L}$ band of SAR images. Journal of Water and Land Development, vol:9, 2005, pp.3-20.

Engman E.T., 2000: Soil moisture. Remote Sensing in Hydrology and Water Management. G. A. Schultz - E. T. Engman Eds. Springer Verlag, Berlin Heidelberg, Germany, 2000, 475 pp.

Kong X. y Dorling S., 2005: The retrieval of soil moisture from medium resolution ENVISAT ASAR wide swath data. Proceedings of the 2004 Envisat \& ERS Symposium, 6-10 September 2004, Salzburg, Osterreich (ESA SP-572, April 2005).

Lievens H. y Verhoest N.E.C., 2012: Spatial and temporal soil moisture estimation from RADARSAT-2 imagery over Flevoland, The Netherlands. Journal of Hydrology 456-457 (2012), pp.44-56.

Moran S., Peters-Lidard Ch., Watts J.y McElroy S., 2004: Estimating soil moisture at the watershed scale with satellite-based radar and land surface models. Can. J. Remote Sensing.,Vol.30, №5, 2004, pp.805-826.

Robock A., Vinnikov K.Y., Srinivasan G., Entin J.K., Hollinger S.E., Speranskaya S.A., Liu S.X. y Namkhai A., 2000: The Global
Soil Moisture Data Bank". Bulletin of the American Meteorological Society, 81(6), 2000. pp.1281-1299.

Salgado H, 2012: Humedad del suelo en cuencas hídricas. Aplicación de herramientas geomáticas y modelización hidrológica. Editorial Académica Española (EAE), AV Akademikerverlag GmbH \& Co. KG, Saarbrücken, Germany, 176 pp.

Salgado H., Génova L., Brisco B. y Bernier M., 2001: Surface soil moisture estimation in Argentina using Radarsat-1 imagery. Canadian Journal of Remote Sensing, 2001, 27(6), pp.685-690.

Santi E., Paloscia S., Pampaloni P., Pettinato S. y Poggi P., 2005: Retrieval of soil moisture from ENVISAT ASAR images: a comparison of inversion algorithms. Proceedings of the 2004 Envisat \& ERS Symposium, 6-10 September 2004, Salzburg, Austria [ESA SP-572, April 2005].

Schmugge T. y J. Wang, 1980: An empirical model for the complex dielectric permittivity of soils as function of water content. IEEE Trans. Geosc. Rem.Sens. Vol.18, pp.288-295.

Touré, A., K.P.B. Thomson, G. Edwards, R.J. Brown y B. Brisco, 1994: Adaptation of the MIMICS backscattering model to the agricultural context: wheat and canola at $\mathrm{L}$ and $\mathrm{C}$ Bands. IEEE transactions of geoscience and remote sensing, 32 (1), 4761.

Ulaby F.T., 1974: Radar measurement of soil moisture content. IEEE Trans. Antennas Propagation. Vol.AP-22(2), pp.257-265.

Ulaby F.T. y Batlivala P.P., 1976: Optimum radar parameters for mapping soil moisture. IEEE transactions geoscience electronics, GE-14 (2), $81-93$.

Ulaby F.T. y Dobson M.C., 1988: Handbook of Radar Scattering Statistics for Terrain. Artech House, 1988.

Ulaby, F.T., Dubois, P. C. y van Zyl, J., 1996: Radar mapping of surface soil moisture. Journal of Hydrology, 184, 57-84.

US Soil Survey Staff, 1975. Soil Taxonomy: A basic system of soil classification for making and interpreting soil maps, US. Department of Agriculture, Agriculture Handbook №436, 
Estimación de la humedad superficial del suelo...

USA, 1975, $754 \mathrm{pp}$,

Wang L. y Qu J.J., 2009: Satellite remote sensing applications for surface soil moisture monitoring: A review. Front. Earth Science, China 2009, 3(2), pp.237-247.
MPIP(O)

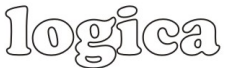

Este es un artículo de acceso abierto distribuido bajo la licencia Creative Commons, que permite el uso ilimitado, distribución y reproducción en cualquier medio, siempre que la obra original sea debidamente citada. 